Journal: Small (Wiley)

\title{
Nanoscale Charge Percolation Analysis in Polymer-Sorted (7,5) Single-Walled Carbon Nanotube Networks
}

Francesca Bottacchi, Stefano Bottacchi, Florian Späth, Imge Namal, Tobias Hertel, and Thomas D. Anthopoulos ${ }^{*}$

F. Bottacchi, Prof. T. D. Anthopoulos

Department of Physics and Centre for Plastic Electronics, Imperial College London, SW7 2BW, London, United Kingdom

E-mail: t.anthopoulos@imperial.ac.uk

Dr. S. Bottacchi

Independent Consultant, via don Gnocchi 33, 20148, Milan, Italy

F. Späth, I. Namal, Prof. T Hertel

Institute of Physical and Theoretical Chemistry, Faculty of Chemistry and Pharmacy, Julius-Maximilian University Würzburg, Am Hubland, 97074 Würzburg, Germany

Keywords: carbon nanotubes; transistors; network percolation; conductive atomic force microscopy; surface characterization

\begin{abstract}
The current percolation in polymer-sorted semiconducting $(7,5)$ single-walled carbon
\end{abstract} nanotube (SWNT) networks, processed from solution, is investigated using a combination of electrical field-effect measurements, atomic force microcopy (AFM) and conductive AFM (CAFM) techniques. From AFM measurements, the nanotube length in the as-processed $(7,5)$ SWNTs network is found to range from $\sim 100 \mathrm{~nm}$ to $\sim 1500 \mathrm{~nm}$, with a SWNT surface density well above the percolation threshold and a maximum surface coverage $\approx 58 \%$. Analysis of the field-effect charge transport measurements in the SWNT network using a two-dimensional homogeneous random network stick-percolation model yields an exponent coefficient for the transistors OFF currents of 16.3. This value is indicative of an almost ideal random network containing only a small concentration of metallic SWNTs. Complementary C-AFM measurements on the other hand enable visualization of current percolation pathways in the $x y$ 


\section{F. Bottacchi et al. Small (2016), DOI: 10.1002/smll.201600922}

plane and reveal the isotropic nature of the as-spun $(7,5)$ SWNT networks. This work demonstrates the tremendous potential of combining advanced scanning probe techniques with field-effect charge transport measurements for quantification of key network parameters including current percolation, surface coverage and degree of SWNT alignment. Most importantly, the proposed approach is general and applicable to other nanoscale networks, including metallic nanowires as well as hybrid nano-composites.

\section{Introduction}

Over the last several years, semiconducting single-walled carbon nanotubes (SWNTs) have been employed in a wide range of optoelectronic devices, including field-effect transistors ${ }^{[1-6]}$ (FETs), solar cells,${ }^{[7]}$ integrated circuits, ${ }^{[8-10]}$ radio frequency ID (RFID) antennas ${ }^{[11]}$ and lightemitting devices. ${ }^{[12,13]}$ In addition to their attractive electronic properties, SWNTs can be easily solution-processed, hence making them extremely attractive for application in plastic microelectronics. However, most common synthesis methods produce a mixture of nanotube diameters and chiral angles, which typically results in only two-thirds of the as-synthesized SWNTs being semiconducting and the remaining one-third metallic. ${ }^{[14,15]}$ This has a big impact on many opto-electronic applications, and especially in microelectronics, where the presence of metallic SWNTs, even at concentrations of few parts per thousand, could dramatically degrade device performances. ${ }^{[2,15]}$ To overcome this issue, various post-synthetics sorting methods have been recently developed, such as gel chromatography, ${ }^{[16]}$ density gradient ultracentrifugation ${ }^{[17]}$ and polymer wrapping ${ }^{[18-21]}$. Among these methods, polymer wrapping with conjugated polymers, such as polyfluorene-based derivatives, ${ }^{[18-21]}$ is the simplest and most effective way of dispersing and sorting semiconducting SWNTs with specific diameters. Although, on one hand significant progress has been made to optimize sorting and enrichment techniques, on the other 


\section{F. Bottacchi et al. Small (2016), DOI: 10.1002/smll.201600922}

hand the ability to accurately characterize the residual metallic content in highly pure semiconducting samples still remains a major challenge. Raman spectroscopy is one of the more common means to assess metallic content. ${ }^{[22,23]}$ However, here one of our objectives is to demonstrate how the metallic content can be determined by electrical characterization without having to use Raman spectroscopy.

In addition to the residual concentration of metallic SWNTs, other key factors that influence the performance of SWNT FETs are the length of the channel $\left(\mathrm{L}_{\mathrm{C}}\right)$ as compared to the length of the nanotubes $\left(\mathrm{Ls}_{s}\right)$ in the network, the surface density $(\rho)$ of the whole population of SWNTs, and their degree of alignment. ${ }^{[15,24]}$ In fact, for longer channel devices, where $\mathrm{L}_{\mathrm{C}}>\mathrm{L}_{\mathrm{S}}$, high current ON/OFF ratio could be reached even with a small percentage of residual metallic carbon nanotubes (CNTs), as long as they do not form percolating pathways across the channel resulting to electrical shorts between the source and drain electrodes. This can be achieved by keeping the surface density of residual metallic SWNTs as low as possible and ideally below the percolation threshold. This is the situation where percolation theory can then be applied. For short channel devices $\left(L_{C} \leq L_{S}\right)$ the situation is very different because the presence of even a single metallic nanotube could in principle electrically short the source and drain electrodes and drastically reduce the transistor's current $\mathrm{ON} / \mathrm{OFF}$ ratio due to their high current transporting capabilities. ${ }^{[25,26]}$ This case is known as the direct conduction regime. Lowering the density of the SWNT network can help to minimize the impact of metallic SWNTs but it has an adverse effect on charge carrier mobility and channel transconductance. Moreover, even a small degree of alignment between SWNTs could be beneficial on the electronic properties of the network. ${ }^{[15,27]}$ Therefore, achieving simultaneously high channel current ON/OFF ratio and high on- 


\section{F. Bottacchi et al. Small (2016), DOI: 10.1002/smll.201600922}

conductance remains a significant challenge even for the purest solution processed semiconducting SWNT networks reported to date.

Here, we report a new method based on the combination of different characterization techniques, which enables the quantification of residual metallic nanotubes content in a highly pure $(7,5)$ SWNT network. Polymer [poly(9,9-di-n-octylfluorenyl-2,7-diyl)] (PFO) was used to disperse and select the $(7,5)$ semiconducting SWNTs from a mixture of nanotube chirality. Fieldeffect transistors based on a dense random network of polymer-sorted $(7,5)$ SWNTs deposited from solution at room temperature, show primarily p-channel behavior with weak electron accumulation depending on the gate dielectric and device geometry chosen. By using the stickpercolation model, ${ }^{[28,29]}$ the drain current $\left(\mathrm{I}_{\mathrm{D}}\right)$ scaling behavior in the linear operating regime (low $\mathrm{V}_{\mathrm{G}}$ bias) of the transistors as a function of $\mathrm{L}_{\mathrm{C}}$ was analyzed and the residual metallic nanotubes were quantified. The surface topography of the as-processed networks was studied via highresolution atomic force microscope (AFM), whereas the current percolation in the networks was investigated using a variant of the conductive atomic force microscopy (C-AFM) technique. ${ }^{[30-32]}$ The combination of these scanning probe methods allows the direct visualization of the current percolation pathways with nanometer spatial resolution and the simultaneous evaluation of key network properties, such as the resistivity, the degree of alignment (anisotropy), the density of SWNTs and the surface coverage.

\section{Results and Discussion}

\section{1 (7,5) SWNT Dispersion and Morphological Characterization}

The sorted $(7,5)$ SWNTs were dispersed from a mixture of nanotube chirality by exploiting the interaction between the nanotube and the side-chains attached to the PFO backbone. ${ }^{[18]}$ It has 


\section{F. Bottacchi et al. Small (2016), DOI: $\underline{10.1002 / \text { smll.201600922 }}$}

been previously shown that polyfluorene and its derivatives have the ability to selectively wrap and solubilize semiconducting SWNTs with specific diameters. Although the exact selection mechanism is still debated, the number and type of side-chains, the molecular weight of the dispersing polymer, the concentration as well as the viscosity of the solvent seem all to affect the polymers selectivity toward semiconducting SWNTs. ${ }^{[33]}$

Figure 1 shows the absorption spectrum of the polymer-sorted (7,5) SWNTs dispersion in chlorobenzene. The chemical structure of the PFO is shown in the inset. The experimental evidence of the presence of nearly single chirality $(7,5)$ SWNTs arises from the existence of two sharp absorption peaks at $1050 \mathrm{~nm}$ and $655 \mathrm{~nm}$, which correspond respectively to the first $\left(\mathrm{E}_{11}\right)$ and second $\left(\mathrm{E}_{22}\right)$ excitonic sub-band transitions of the $(7,5)$ SWNT. ${ }^{[20]}$ Two additional smaller peaks are visible at $995 \mathrm{~nm}$ and $1140 \mathrm{~nm}$, which may be due to residual amounts of $(6,5)$ and $(7,6)$ semiconducting SWNTs, respectively. ${ }^{[1,21]}$ From Figure 1, the carbon-atom concentration of the $(7,5),(6,5)$ and $(7,6)$ nanotube species can be found, from which we can obtain the following relative fractions: $(7,5) \approx 82 \%,(6,5) \approx 8.9 \%$, and $(7,6) \approx 9.1 \% .{ }^{[34,35]}$ Most importantly, there is no significant absorption in the range of $450 \mathrm{~nm}-550 \mathrm{~nm}$, which is where absorption from metallic SWNTs in the $(7,5)$ diameter range would appear, ${ }^{[18,20,21,36]}$ clearly highlighting the effectiveness of using PFO as the dispersant polymer. However, an exact quantification of the possible residual amount of metallic SWNTs from the absorption spectrum is difficult due to background absorption and to the very weak intensity in that range that is comparable to the noise level of our instrument. Finally, the features in the range of $340 \mathrm{~nm}-$ $380 \mathrm{~nm}$ correspond to the absorption of the polymer PFO. Thus, on the basis of these results, we expect polymer-sorted $(7,5)$ SWNT networks to behave as intrinsic semiconductors with similar hole and electron mobility. ${ }^{[1,4,5]}$ 


\section{F. Bottacchi et al. Small (2016), DOI: 10.1002/smll.201600922}

The surface morphology of the as-spun $(7,5)$ SWNT networks was studied by tapping-mode atomic force microscopy (AFM). Figure 2(a) shows the AFM image of the surface topography of a $(7,5)$ SWNT network deposited onto $\mathrm{SiO}_{2}$, while Figure 2(b) displays a magnified area of the same network where some larger bundles/nanotube aggregates are clearly visible. SWNTs appear to be randomly distributed over the entire $\mathrm{SiO}_{2}$ surface without a preferential orientation. The square-length weighted abundance distribution was used to estimate the effective length contributing to percolation, in order to account for the bias of percolation towards longer nanotubes in a sample with length-dispersion. Using the AFM image in Figure $\mathbf{S 2}^{[37]}$ and the histogram of lengths in Figure S3, ${ }^{[37]}$ the effective nanotube length (Ls) was estimated to be 680 $\mathrm{nm}$, with a surface density well above the percolation threshold of $12 \mathrm{SWNT} \mu \mathrm{m}^{-2}\left(\rho_{t h}=\right.$ $\left.4.236^{2} / \pi \mathrm{Ls}^{2}\right),{ }^{[25]}$ and thus the carrier mobility can be extracted using the parallel plate capacitor model within the gradual channel approximation. ${ }^{[38]}$ Further details on the CNT length characterization can be found in the Supporting Information. ${ }^{[37]}$ Further information about the network morphology arises from the surface coverage of the $(7,5)$ SWNT network, and it is provided by the height counts distribution and the height cumulative distribution shown in Figure 2(c). Because SWNTs are deposited onto $\mathrm{SiO}_{2}$, the total height counts distribution (blue line) corresponds to the convolution of the independent height counts distributions respectively of $\mathrm{SiO}_{2}$ (red line) and $(7,5)$ SWNTs.

Based on this assumption, and by assuming a (7,5) SWNT diameter (d) of $0.82 \mathrm{~nm},{ }^{[20,21]}$ we combined the properties of the convolution theory and the height cumulative distribution [green line in Figure 2(c)] to obtain the following coverage percentages: $(7,5)$ SWNTs cover $\approx 58 \%$ of the total surface area scanned, $\mathrm{SiO}_{2}$ covers $\approx 42 \%$. We note however that these values are only 


\section{F. Bottacchi et al. Small (2016), DOI: 10.1002/smll.201600922}

indicative of the upper limit for the SWNTs coverage and not an accurate estimation due to the following reasons: (i) the AFM probe has an atomic-scale vertical resolution and a lateral resolution comparable to the probe-radius so, the higher the resolution of the scan, the more accurate the extracted coverage value is; (ii) the residual PFO present in the network most likely surrounds the nanotubes and potentially increases their apparent diameter; (iii) small amounts of residual $(6,5)$ and $(7,6)$ SWNTs with different diameters from the $(7,5)$ species are also known to exist in our samples, providing some perturbation in the estimation of the single chirality SWNT coverage; and (iv) this coverage value refers to the total amount of material deposited on $\mathrm{SiO}_{2}$ and thus it includes carbon nanotubes, carbon nanotube bundles, carbon aggregates, and polymers. Despite of these relatively minor issues, our approach results in a simple and rapid way for quantifying the substrate surface coverage of a given nano-material distribution using the surface topography obtained by conventional AFM.

\section{$2.2(7,5)$ SWNT Field-Effect Transistors}

Top-gate, bottom-contact field-effect transistors [Figure 3(a)] were fabricated by spin-coating the polymer-sorted $(7,5)$ SWNT dispersion on glass substrates containing the thermally evaporated source (S) and drain (D) electrodes. Device fabrication was completed with the spincoating of a Cytop layer acting as the gate dielectric and the deposition of the top Al gate electrode via thermal sublimation in high vacuum $\left(10^{-6} \mathrm{mbar}\right)$. Figure 3(b) and Figure 3(c) show representative sets of the transfer and output characteristics, respectively, measured for a solution-processed top-gate FET with $\mathrm{L}_{C} / \mathrm{W}_{\mathrm{C}}=50 \mu \mathrm{m} / 1000 \mu \mathrm{m}$, where $\mathrm{W}_{\mathrm{C}}$ is the channel width. The device exhibits strong ambipolar charge transport characteristics ${ }^{[1,4,5]}$ with balanced hole and electron currents and a channel current ON/OFF ratio of $\sim 10^{4}$. The gate leakage current was always found to be at least two orders of magnitude lower than the channel ON current, while the 


\section{F. Bottacchi et al. Small (2016), DOI: $\underline{10.1002 / \text { smll.201600922 }}$}

small operating hysteresis is attributed to atmospheric oxidants/adsorbates that are present even within the nitrogen glovebox, albeit at relatively small concentrations ( ppm). ${ }^{[39,40]}$ Finally, the resulted electron and hole mobility values extracted are $\sim 0.03 \mathrm{~cm}^{2} \mathrm{~V}^{-1} \mathrm{~s}^{-1}$ and $\sim 0.02 \mathrm{~cm}^{2} \mathrm{~V}^{-1} \mathrm{~s}^{-1}$ respectively.

The charge transport properties of the as-spun $(7,5)$ SWNT networks were also characterized using a bottom-gate, bottom-contact transistor architecture fabricated on $\mathrm{Si}^{++} / \mathrm{SiO}_{2}(400 \mathrm{~nm}$ thick) wafers [Figure 4(a)]. Figure 4(b) and Figure 4(c) show representative sets of the transfer and output characteristics, measured for a transistor with $\mathrm{LC}_{\mathrm{C}} / \mathrm{W}_{\mathrm{C}}=20 \mu \mathrm{m} / 10000 \mu \mathrm{m}$. Unlike the top-gate transistors of Figure 3, this device exhibits a strong p-channel behavior. This can be explained by the presence of electron traps at the $\mathrm{SiO}_{2} /(7,5) \mathrm{SWNT}$ interface and the predominant hole-injecting nature of both $\mathrm{S}$ and $\mathrm{D}$ electrodes due to the high work function of $\mathrm{Au}(\sim 5 \mathrm{eV})$. Previous reports ${ }^{[39-41]}$ have in fact shown that n-type conduction is suppressed in most organic semiconductor-based transistors due to charge traps from the $\mathrm{SiO}_{2}$ interface and especially due to charge transfer to the oxygen/water layer that is strongly bound to the $\mathrm{SiO}_{2}$ surface, where the $\mathrm{H}_{2} \mathrm{O} / \mathrm{O}_{2}$ redox couple occurs. This is particularly important for SWNTs since the valence band position of the $(7,5) \mathrm{SWNT}\left(\mathrm{E}_{\mathrm{V}} \approx-5.2 \mathrm{eV}\right)$ lies very close to the redox potential of oxygen $\left(\mathrm{E}_{\mathrm{REDOX}} \approx-5.3 \mathrm{eV}\right)$ dissolved in slightly acidic water $(\mathrm{pH}=6)$ adsorbed on the $\mathrm{SiO}_{2}$ surface. For this reason, electrons will efficiently transfer from the $(7,5)$ SWNT network to the oxygen/water layer. ${ }^{[39]}$ This electrochemical charge transfer process is also likely to generate negative chemical intermediates, which in turn create and charge acceptor states on the substrate surface. ${ }^{[39]}$ These surface states may then affect the operating hysteresis that is often seen in bottom-gate SWNTbased transistors made on bare $\mathrm{SiO}_{2}$. In addition to this, another possible cause for the hysteresis is the presence of residues of the cellulose membrane used to filter the nanotubes during the 


\section{F. Bottacchi et al. Small (2016), DOI: $\underline{10.1002 / \text { smll.201600922 }}$}

polymer-sorting procedure (see Experimental Section). Despite of these non-idealities, the device exhibits good performances with a current ON/OFF ratio $>10^{6}$, a gate leakage two orders of magnitude lower than the $\mathrm{ON}$ current, a hole mobility of $\sim 0.24 \mathrm{~cm}^{2} \mathrm{~V}^{-1} \mathrm{~s}^{-1}$ and well visible current saturation behavior.

Finally, we remark that both the top-gate and bottom-gate $(7,5)$ SWNT transistors shown in Figure 3 and Figure 4, respectively, have not been optimized for achieving high carrier mobility and low-voltage operation, but have been primarily used to study the impact of the device geometry on charge transport and for the current percolation analysis. Moreover, due to the nonuniform substrate surface coverage provided by the $(7,5)$ SWNTs, the channel width that effectively take part to the transport phenomena is smaller than the geometrical width that has been used to evaluate the carrier mobility, leading to an underestimated value for both transistor geometries. A more accurate description that takes into account the realistic electrostatic coupling between carbon nanotubes and the gate electrode through the quantum capacitance of SWNTs can be used to estimate the gate capacitance of SWNT transistors with higher precision. ${ }^{[42]}$ By applying this model, the gate capacitance reduces while the charge carrier mobility increases. Although of renowned relevance, such analysis is beyond the scope of this work, as its contribution would be negligible, and will be the subject of a future study.

\subsection{Analysis of the Current Percolation using Field-Effect Transistors}

The current scaling behavior as a function of channel length $\left(\mathrm{L}_{\mathrm{C}}\right)$ for different bottom-gate $(7,5)$ SWNT-based transistors [Figure 4(a)] was also investigated. Figure 5 shows the transfer characteristics measured at the constant drain-source voltage $V_{D}=-10 \mathrm{~V}$ for three transistors with $\mathrm{L}_{\mathrm{C}}=2 \mu \mathrm{m}, 10 \mu \mathrm{m}$, and $40 \mu \mathrm{m}$, fabricated on different substrates. While the channel ON 


\section{F. Bottacchi et al. Small (2016), DOI: 10.1002/smll.201600922}

current in all devices decreases with increasing channel length, due to the corresponding reduction of the electric field along the channel, the channel OFF current is also found to increase drastically with decreasing $\mathrm{L}_{\mathrm{C}}$. The latter observation suggests that charge transport in the transistor channel is dominated by the percolation of metallic SWNTs and is particularly pronounced in devices with shorter $L_{C}$. A further important observation is the presence of device-to-device variability, which is manifested as a deviation of the $\mathrm{ON}$ current dependence on $\mathrm{L}_{\mathrm{C}}$ from the ideal Ohmic behavior (Figure 5). There are several possible reasons for this observation including: (i) the presence of excess polymer and/or residues of the cellulose membrane in the SWNT dispersion, which degrade device performance as it reduces the maximum channel ON current; (ii) fluctuations in the oxygen concentration inside the glovebox, which is known to act as p-dopant for SWNTs; and (iii) the random nature of the spin-coated SWNTs networks. Due to the latter characteristic, in particular, even devices with the same channel dimensions prepared under the same conditions on different substrates will appear to behave slightly differently. This is why the channel current in transistors fabricated on different substrates (e.g. Figure 5) deviate from the ideal Ohmic behavior. In an effort to avoid such unwanted effects, channel current scaling behaviour analysis (Section 2.3.2) was performed using devices made on the same substrates/batches.

\subsubsection{Two-Dimensional Homogeneous Random-Network Stick Percolation Model}

In order to study the charge transport properties in the percolation regime of our transistors and to estimate the metallic content in the channel SWNT networks, we applied the stickpercolation model for an ideal 2D random-network developed by Kumar et al., ${ }^{[24]}$ and Kocabas $e t$ al. ${ }^{[25]}$ for CVD-grown CNT networks with a known surface density and metallic content. For this purpose, we considered device operation in the linear regime (low bias) and assumed a constant 


\section{F. Bottacchi et al. Small (2016), DOI: $\underline{10.1002 / \text { smll.201600922 }}$}

charge density along the channel. This approximation is known as the constant conductivity approximation and the transport properties of the $(7,5)$ SWNT channels can be described by the two-dimensional (2D) homogeneous random-network stick-percolation model. ${ }^{[28]}$ The universal current-scaling equation for $\mathrm{I}_{\mathrm{D}}$ in the low-bias regime reduces to: ${ }^{[25]}$

$$
\mathrm{I}_{\mathrm{D}} \cong \frac{\mathrm{k}}{\mathrm{L}_{\mathrm{S}}}\left(\frac{\mathrm{L}_{\mathrm{S}}}{\mathrm{L}_{\mathrm{C}}}\right)^{m}
$$

where $\mathrm{k}(\mathrm{A} \mathrm{cm})$ depends on the stick to stick interaction $\left(\mathrm{C}_{\mathrm{i}, \mathrm{j}}\right)$, the nanotube diameter $(\mathrm{d})$, the oxide capacitance $\left(\mathrm{C}_{\mathrm{Ox}}\right)$, the channel width $\left(\mathrm{W}_{\mathrm{C}}\right)$ and bias conditions, while the exponent $m$ depends on the normalized coverage $\left(\rho \mathrm{Ls}^{2}\right)$ and the anisotropy of the nanotubes.

In the case of a random network with a normalized coverage much higher than the percolation threshold $\left(\rho \mathrm{Ls}^{2} \gg 4.236^{2} / \pi\right),{ }^{[25]}$ most of the sticks are connected together forming several conductive paths and therefore the network behaves as a 2D conductor, with $m \sim 1$. Thus, in the limit of the $2 \mathrm{D}$ conductor, the universal current-scaling formula reduces to the well-known Ohm's law, where $\mathrm{I}_{\mathrm{D}} \sim \mathrm{k} / \mathrm{L}_{\mathrm{C}}$. Vice versa, if the normalized coverage is below the percolation threshold $\left(\rho_{t h} \mathrm{Ls}_{S}^{2}=4.236^{2} / \pi\right)$, most of the sticks are not interconnected and the presence of conducting paths between the drain and source electrodes strongly depends on the channel length $\left(\mathrm{L}_{\mathrm{C}}\right)$ as compared to the length of the sticks $\left(\mathrm{Ls}_{\mathrm{S}}\right)$. This is the case where the percolation regime dominates the channel conduction mechanism, and the exponent $m>1$ can be found experimentally.

\subsubsection{Analysis of the Charge Transport Percolation in (7,5) SWNT Transistors}

On the basis of the previous considerations, we used the channel ON and OFF currents of different $(7,5)$ SWNT transistors to estimate the current exponent $m$ in the ON and OFF states. To 


\section{F. Bottacchi et al. Small (2016), DOI: 10.1002/smll.201600922}

this purpose, we plotted the drain currents in the ON and OFF states of SWNT transistors with constant channel widths $\mathrm{W}_{\mathrm{C}}=10000 \mu \mathrm{m}$ as a function of the channel length $\mathrm{L}_{\mathrm{C}}$, measured in the linear regime $\left(\mathrm{V}_{\mathrm{D}}=-10 \mathrm{~V}\right)$ and at the same $\mathrm{V}_{\mathrm{G}}$. By combining Equation 1 with the experimentally determined ON currents, we were able to extrapolate the current exponent $m_{o n}$, corresponding to the total SWNT network. On the other hand, by analyzing the OFF currents and by using Equation 1, we extrapolated the current exponent $m_{\text {off }}$, corresponding to the percolation of only metallic nanotubes (m-SWNTs). Because of the percolation regime established by the residual metallic SWNTs, we expect that $m_{\text {off }}>m_{o n}$. Based on the same analysis, we were also able to estimate the surface density of the metallic SWNTs $\left(\rho_{m e t}\right.$ in m-SWNT $\left.\mu \mathrm{m}^{-2}\right)$.

Figure 6(a) shows the schematics of the SWNT random networks formed between two Au electrodes for three different conduction regimes (I, II, III). Here black sticks represent the mSWNT while grey sticks represent the semiconducting $(7,5)$ SWNTs. The direct conduction regime (I) is achieved when the channel length is shorter than the length of the semiconducting SWNTs, i.e. $\mathrm{L}_{\mathrm{C}} \leq \mathrm{L}_{\mathrm{S}}$, so the presence of even a single (or a few) SWNTs can lead to the formation of a direct electrical connection. If $\mathrm{m}-\mathrm{SWNT}$ s are also present in the network, they could form electrical shorts across the channel, shunting the semiconducting SWNTs and drastically increasing the OFF current, independently from any applied gate potential. In the direct conduction regime (I), the total current that flows across the device in both $\mathrm{ON}$ and OFF states results inversely proportional to the channel length, as shown in Figure 6(b). We remark that using logarithmic scale on both axes, the drain current decreases linearly with the channel length, confirming the expected behavior of the Ohm's law. In regime (II), where the channel length is relatively longer than the stick length, $\mathrm{L}_{\mathrm{C}}>\mathrm{L}_{\mathrm{S}}$, the conduction in the OFF state is dominated by the percolation of the SWNTs. However, even if m-SWNTs cannot directly bridge 


\section{F. Bottacchi et al. Small (2016), DOI: 10.1002/smll.201600922}

the source and drain electrodes, they could form percolating paths that shunt the percolation paths of the semiconducting SWNTs, thus increasing the OFF current. From regime (II) $m_{\text {on }}$ and $m_{\text {off }}$ can be extracted by extrapolating the linear regions of the $\mathrm{ON}$ and OFF current plotted against Lc. Once $m_{\text {off }}$ and Ls are known, $\rho_{m e t}$ can be calculated. Finally, charge transport in regime (III) is dominated by thermally excited carriers, which are responsible for the non-negligible OFF currents seen in long $\mathrm{L}_{\mathrm{C}}$ devices. We note, however, that measurement limitations associated with the experimental setup and in particular the sensitivity of the instruments might result in an apparent higher value of the OFF channel current, misleading the OFF current vs. channel length behavior with an unexpected background value.

Figure 6(b) displays the $\mathrm{L}_{C}$ dependence of the channel $\mathrm{ON}$ and $\mathrm{OFF}$ currents and the corresponding fittings (dash lines) using $m_{o n}=1.02$ and $m_{o f f}=16.3$. In order to account for the observed device-to-device variability (Figure 5) and also to provide a more robust and consistent analysis, four transistors per $\mathrm{L}_{\mathrm{C}}$ dimension coming from the same substrate batch have been characterized under the same conditions, and their ON and OFF currents have been averaged. The ON current [Figure 6(b), black line] exhibits an inversely proportional dependence on $\mathrm{L}_{\mathrm{C}}$ and it is attributed to the dense distribution of the SWNT network whose conductive behavior is well approximated by the 2D Ohmic conductor limit $(m \sim 1)$. In contrast, the OFF current is found to reduce by six orders of magnitude from $\sim 3 \mathrm{~mA}$ to $\sim 0.3 \mathrm{nA}$ within the $\mathrm{L}_{\mathrm{C}}$ range of $2-5$ $\mu \mathrm{m}$ (the straight line with constant negative slope in logarithmic scale). The latter $\mathrm{L}_{\mathrm{C}}$ range $(2-5$ $\mu \mathrm{m}$ ) should correspond to the percolation regime (II) in Figure 6(a) which, as already discussed, is dominated by SWNTs percolation. These findings are in excellent agreement with the experimentally measured average nanotube length (Figure S2), since in this regime $\mathrm{L}_{\mathrm{C}}$ must be longer than Ls. Also, the fact that $m_{\text {off }}=16.3$, indicates that a nearly ideal condition is reached 


\section{F. Bottacchi et al. Small (2016), DOI: 10.1002/smll.201600922}

$\left(\rho_{\text {met }}<\rho_{t h}\right)$, corresponding to a very low surface density of metallic SWNTs present in the channel. The $\mathrm{L}_{\mathrm{C}}$ dependence of the current ON/OFF ratio is also shown in Figure 6(c), where it ranges from a value of 1 , for $\mathrm{L}_{C}<2 \mu \mathrm{m}$, i.e. regime (I), up to $3.5 \times 10^{6}$ for $\mathrm{L}_{\mathrm{C}}>5 \mu \mathrm{m}$, i.e. regime (III).

By using previously published experimental results obtained with CVD-grown carbon nanotubes by Kocabas et al. ${ }^{[25]}$ and Sun et al., ${ }^{[26]}$ we have plotted the curve of the universal current exponent $m$ by fitting their experimental data, as shown in Figure 6(d). Then, by using $m_{o n}=1.02, m_{o f f}=16.3$, and a value for $\mathrm{Ls}_{\mathrm{s}}$ of $680 \mathrm{~nm}$, we estimated both the total surface density $(\rho)$ and the surface density of metallic SWNTs $\left(\rho_{m e t}\right)$ to be approximately $184 \mathrm{SWNT}_{\mu \mathrm{m}^{-2}}$ and $4.3 \mathrm{~m}-\mathrm{SWNT} \mu \mathrm{m}^{-2}$ respectively, with a correspondent metallic percentage of $2.3 \%$. Choosing different values for $\mathrm{L}_{\mathrm{s}}$ from the experimentally determined range of $100 \mathrm{~nm}$ and $1500 \mathrm{~nm}$ (close to the limits of the SWNT length distribution seen in Figure S2) yields values for $\rho$ of 2125 SWNT $\mu \mathrm{m}^{-2}$ and $85 \mathrm{SWNT} \mu \mathrm{m}^{-2}$, and for $\rho_{m e t}$ of $50 \mathrm{SWNT} \mu \mathrm{m}^{-2}$ and $2 \mathrm{SWNT} \mu \mathrm{m}^{-2}$, respectively for Ls of $200 \mathrm{~nm}$ and $1000 \mathrm{~nm}$. While the surface density of carbon nanotubes strongly depends on the actual length considered for the calculations, the percentage of metallic nanotubes instead is independent from $\mathrm{LS}_{\mathrm{S}}$, and depends only on the $m_{o n}$ and $m_{\text {off }}$ values obtained through transistor characterization. Thus, we can conclude that the metallic percentage of our $(7,5)$ SWNT dispersion is approximately $2.3 \%$, even if the SWNTs are broadly distributed in length.

However, the presence of the polymer in our network certainly affects the value of the parameter $\mathrm{k}$ in the current-scaling Equation 1 with respect to the one obtained without any polymer. Moreover, the nanotubes in our network are not rigid sticks but they are flexible, with some defects, they interact with each other and with the substrate, forming bundles and 


\section{F. Bottacchi et al. Small (2016), DOI: $\underline{10.1002 / \text { smll.201600922 }}$}

aggregates, and $L_{S}$ is broadly distributed. For these reasons the above surface densities and metallic percentage must be considered only as indicative values. In any case, we conclude consistently that $\rho_{\text {met }}$ is very small compared to the total $(7,5)$ SWNTs surface density. However, such tiny amount of metallic SWNTs, over a channel width of $10000 \mu \mathrm{m}$, is still able to completely shunt almost all devices with $\mathrm{L}_{\mathrm{C}} \leq 2 \mu \mathrm{m}$, dramatically reducing the current ON/OFF ratio. The small amount of $\mathrm{m}-\mathrm{SWNT}$ also justifies the absence of any evidence in the absorption spectra in Figure 1. A possible explanation for the lack of any measurable spectrum is that the effective surface density of m-SWNTs is probably lower than the value the percolation theory suggests. To this end, the presence of bundled semiconducting nanotubes in the transistor channel may well contribute to enhance the OFF currents measured. ${ }^{[2,26]}$ However, further work is required in order to prove or refute this hypothesis. Despite this, we have shown that by combining electrical field-effect measurements with the 2D homogeneous random network stickpercolation model, we are able to estimate the surface density of metallic nanotubes. Most importantly, the proposed approach is simple and it could potentially allow quantification of the metallic content of any semiconducting material disposed in a 2D random network and it should therefore be applicable to other material systems described by the same percolation model.

\subsection{Percolation Study through Lateral Conductivity Measurements by Atomic Force Microscopy}

In order to further characterize the percolation behavior of the $(7,5) \mathrm{SWNT}$ network, lateral conductivity measurements using atomic force microscopy (C-AFM), were performed. ${ }^{[30]}$ This technique consists in scanning the active layer's surface deposited over an insulating substrate, with a platinum-coated $(\mathrm{Pt})$ conductive AFM probe in contact, at a known lateral distance from the second gold $(\mathrm{Au})$ electrode. By applying a fixed bias between the C-AFM probe and the Au 


\section{F. Bottacchi et al. Small (2016), DOI: 10.1002/smll.201600922}

electrode, the simultaneous acquisition of both the $(7,5)$ SWNT network topography and the lateral current is possible. In the simplest case, the applied electric field is expected to be inversely proportional to the distance between the scanning C-AFM probe and the remote $\mathrm{Au}$ electrode. However, since the current flow between any two points across the SWNT network depends strongly on its morphology and the various resistive paths (inter/intra-CNT transport, CNT-CNT junctions etc.), the effective electric field is also expected to deviate from its theoretical value. These transport paths are made from a random combination of many semiconducting SWNTs and few metallic ones, which are randomly positioned within the semiconducting population and unfortunately completely hidden from direct visualization via lateral C-AFM. Figure 7(a) shows a schematic representation of the lateral C-AFM setup employed in this study.

Figure 7(b) shows the topographic image of the as-spun polymer-sorted $(7,5)$ SWNT network deposited onto the $\mathrm{SiO}_{2}$ substrate, while Figure 7(c) shows the corresponding resistance $(R)$ map calculated for each $(x, y)$ position of the probe from the measured current map $I(x, y)$ and the applied voltage between the C-AFM probe and the Au electrode. As can be seen in Figure 7(c), only certain nanotubes, close to the Au electrode (schematically depicted at the top of the image but not seen in the $R$ map), are well visible in the network (darker regions). The resistance (current) increases (decreases) as the probe moves away perpendicularly from the Au electrode in the $y$-direction, almost independently from the $x$ position, thus showing a preferred current transport direction perpendicularly to the Au electrode. This is not surprising since the modulus of the electric field reduces proportionally to the distance between the probe and the Au electrode, for the applied constant voltage. This is not the case when the C-AFM probe moves in the $x$-direction (parallel to the electrode), where the electric field remains almost constant and the 


\section{F. Bottacchi et al. Small (2016), DOI: 10.1002/smll.201600922}

current does not change as well. However, we remark that this simplified description is valid in the central region of the substrate, away from the edges of the Au electrode, where the geometry of the electrode determines a non-uniform electric field distribution and border effects take place.

Figure 7(d) shows the resistance distribution $R(x, y)$ of the complete population, where $\mathrm{R}_{\mathrm{AV}}$ is the average value $\left(\mathrm{R}_{\mathrm{AV}} \sim 18 \mathrm{G} \Omega\right)$ and $\mathrm{R}_{\mathrm{STD}}$ is the standard deviation $\left(\mathrm{R}_{\mathrm{STD}} \sim 15 \mathrm{G} \Omega\right)$. It is important to remark that both the resistance $R(x, y)$ and the current $I(x, y)$ function shown in Figure 7(c), strongly depend from the shape of the Au electrode and the position of the scanning C-AFM probe respect to it. For these reasons, both the current and the resistance are not representative of the $(7,5) \mathrm{SWNT}$ network. In order to evaluate the intrinsic electrical properties of the SWNT network, we have calculated the network resistivities $\rho_{\mathrm{x}}(x, y)$ and $\rho_{\mathrm{y}}(x, y)$, defined respectively along $x$ and $y$ directions. Figure 7(e) shows the resistivity distributions together with the corresponding mean and standard deviation values [in $\Omega \mathrm{cm}$ ]. The resistivities $\rho_{\mathrm{x}}(x, y)$ and $\rho_{\mathrm{y}}(x, y)$ have been obtained from the following expressions:

$$
\begin{aligned}
& \rho_{x}(x, y)=\frac{\left|\Delta R(x, y)_{x}\right|}{\Delta x} t(x, y) \Delta y \\
& \rho_{y}(x, y)=\frac{\left|\Delta R(x, y)_{y}\right|}{\Delta y} t(x, y) \Delta x
\end{aligned}
$$

In Equation 2(a) and Equation 2(b), $R(x, y)$ is the resistance at each sampled position, $\Delta \mathrm{x}$ and $\Delta y$ are the $x$ and $y$ measurement steps of the AFM probe, and $t(x, y)$ is the topographic height at the sampled position, that is acquired by the C-AFM simultaneously with the resistance $R(x, y)$. The main advantage of using the $x$ and $y$ resistivity is that they are intrinsic parameters of the network, as they depend only on carbon nanotube network distribution and do not depend from the Au electrode and the applied voltage. In the case of a fully aligned SWNT network along a certain direction, because of different transport mechanisms along the nanotube axis (ballistic- 


\section{F. Bottacchi et al. Small (2016), DOI: $\underline{10.1002 / \text { smll.201600922 }}$}

type) and transverse to the nanotube axis (hopping-type), the resistivity measured along the nanotube axis is characterized by a very narrow distribution, with low average value and low standard deviation. On the other hand, the resistivity in the perpendicular direction (transverse to the SWNT axis) is characterized by a broader distribution, showing both higher average value and standard deviation.

The distributions of the resistivities $\rho_{\mathrm{x}}(x, y)$ and $\rho_{\mathrm{y}}(x, y)$ are shown in Figure 7(e). As expected, they exhibit very similar average values $\left(\rho_{\mathrm{x}, \mathrm{av}}=0.27 \Omega \mathrm{cm}, \rho_{\mathrm{y}, \mathrm{av}}=0.23 \Omega \mathrm{cm}\right)$ and standard deviations $\left(\rho_{\mathrm{x}, \mathrm{std}}=0.62 \Omega \mathrm{cm}, \rho_{\mathrm{y}, \mathrm{std}}=0.57 \Omega \mathrm{cm}\right)$. Accordingly, we can conclude that the measured polymer-sorted $(7,5)$ SWNT network is characterized by an isotropic distribution, showing no preferential orientation (no alignment). Further details about carbon nanotube orientation can be found in the Supporting Information, where a partially aligned blade-coated SWNT film is analyzed. ${ }^{[37]}$ Although this is likely expected for a spin-coated network, it validates the applicability of the 2D random-network model used for the analysis of charge percolation behavior.

\section{Conclusions}

In conclusion, we have reported a novel approach for studying the current percolation behavior in solution-deposited semiconducting 2D-networks composed of polymer-sorted $(7,5)$ SWNTs. The method relies on the combination of AFM topography, electrical field-effect transistors and lateral C-AFM measurements. AFM analysis of as-spun networks was used to fully characterize the $(7,5)$ SWNT surface morphology in terms of length, surface density, and surface coverage. By analyzing the electrical field-effect measurements using the $2 \mathrm{D}$ homogeneous randomnetwork stick-percolation model, we obtained the current exponent for the channel OFF current 


\section{F. Bottacchi et al. Small (2016), DOI: 10.1002/smll.201600922}

to be as high as 16.3. The electrical measurements also revealed the presence of three conduction regimes depending on $\mathrm{L}_{\mathrm{C}}$. Regime (I) was identified as the direct conduction regime and was observed for devices with $\mathrm{L}_{\mathrm{C}}<2 \mu \mathrm{m}$. Regime (II) was identified as the percolation conduction regime and was found to dominate charge transport in transistors with $2 \mu \mathrm{m}<\mathrm{L}_{\mathrm{C}}<5 \mu \mathrm{m}$. Finally, regime (III) was attributed to the transport of thermally excited carriers and was observed only in transistors with $\mathrm{L}_{\mathrm{C}}>5 \mu \mathrm{m}$. Through the use of the lateral $\mathrm{C}$-AFM technique, we were also able to map the current percolation pathways in the SWNT network with nanoscale spatial accuracy. These measurements allowed calculation of the resistivity distributions in the $(7,5)$ SWNT network as a function of $(x, y)$ position while verifying its isotropic nature. The proposed method is new and powerful, and it can be used for the analysis of surface coverage, metallic content, degree of alignment and charge percolation behavior in any given 2D semiconducting network.

\section{Experimental Section}

Preparation of Polymer-Sorted (7,5) SWNT Dispersion: $100 \mathrm{mg}$ of single-walled carbon nanotubes (Sigma-Aldrich, CoMoCat SWNTs, diameter $0.7 \mathrm{~nm}-0.9 \mathrm{~nm}$, SWNT content $\geq 77$ $\%$ ) were dispersed in $200 \mathrm{ml}$ of toluene together with $360 \mathrm{mg}$ of polyfluorene [poly(9,9-di-noctylfluorenyl-2,7-diyl)] [PFO, Figure 1 inset] (Sigma-Aldrich, $\mathbf{M}_{\mathrm{w}} \geq 20000$ ). The mixture was then subjected to ultrasonication using a horn sonicator for 14 hours consecutive. Subsequently, the dispersion was centrifuged at $4000 \mathrm{rpm}$ for 30 minutes and the supernatant was carefully selected. ${ }^{[21]}$ The excess polymer was then removed via vacuum filtration and the resulting $(7,5)$ SWNTs were collected on a cellulose membrane. Finally, the membrane was dissolved in acetone and the resulting SWNTs, floating on the acetone surface, were re-dispersed in chlorobenzene through 20 minutes ultra-sonication and 1 hour cup sonication. The (7,5) SWNT dispersion was 


\section{F. Bottacchi et al. Small (2016), DOI: $\underline{10.1002 / \text { smll.201600922 }}$}

then characterized by absorption spectroscopy with a Varian Cary 5000 UV-Vis-NIR spectrophotometer in absorbance mode, with $1 \mathrm{~mm}$ light path cuvette and background correction for chlorobenzene absorbance.

Transistor Fabrication: Top-gate, bottom-contact devices were fabricated on glass with thermally evaporated $5 \mathrm{~nm} / 40 \mathrm{~nm}$ of $\mathrm{Al} / \mathrm{Au}$ source and drain (S/D) contacts. The Al underlayer provides improved adhesion to the glass, and does not affect charge injection because in this particular top-gate, bottom-contact transistor architecture the charge transporting channel is in direct contact with the top Au layer of the bilayer S/D electrodes. The lower surface (Al) is in contact with the glass and it does not contribute to any charge injection. The $(7,5)$ SWNT dispersion was ultra-sonicated for 30 minutes prior to deposition to ensure that the nanotubes were well dispersed. Spin coating was carried out in a nitrogen atmosphere at $1000 \mathrm{rpm}$ for 30 seconds, followed by 15 minutes annealing at $90{ }^{\circ} \mathrm{C}$ to remove residual solvent. Then, $900 \mathrm{~nm}$ of the fluoropolymer gate dielectric $\mathrm{CYTOP}^{\circledR}$ were spin casted at $2000 \mathrm{rpm}$ for 1 minute onto the semiconductor surface and annealed at $100{ }^{\circ} \mathrm{C}$ for 20 minutes before $40 \mathrm{~nm}$ Al gate electrodes were deposited by thermal evaporation. Because SWNT films are not perfectly uniform, it is possible that $\mathrm{CYTOP}^{\circledR}$ partially infiltrates the network forming a conformal coating around the CNTs, hence resulting to a stronger capacitive coupling of the gate to the channel. However, the latter effect is considered unlikely, or insignificant, since CYTOP ${ }^{\circledR}$ is very viscous and forms thick layers. To avoid any such issues however, top-gate transistors have not been used for the percolation analysis, hence eliminating such unwanted effects. Bottom-gate, bottom-contact devices composed of doped $\mathrm{Si}^{++}$wafer as the gate electrode, a thermally grown $200 \mathrm{~nm}$-thick layer of $\mathrm{SiO}_{2}$ as the gate dielectric and pre-patterned $10 \mathrm{~nm} / 50 \mathrm{~nm} \mathrm{Ti} / \mathrm{Au}$ source and drain electrodes, were used for the study of charge percolation. As-prepared materials were then deposited directly on the bottom-gate, bottom contact transistor structures. The procedure for the 


\section{F. Bottacchi et al. Small (2016), DOI: 10.1002/smll.201600922}

$(7,5)$ SWNT dispersion deposition is the same as for the top-gate FETs. For samples studied by lateral C-AFM, the source/drain electrodes were then contacted with silver paste and copper wire to connect with the atomic force microscope. Bottom-gate bottom-contact transistors on $\mathrm{Si}^{++} / \mathrm{SiO}_{2}$ with $\mathrm{L}_{\mathrm{C}}$ of $0.75,1,1.25,1.5,2,2.5,3,5,7.5,10,20,30$, and $40 \mu \mathrm{m}$ and $\mathrm{W}_{\mathrm{C}}$ of 10000 $\mu \mathrm{m}$, were used to study the current scaling behavior. All devices have been fabricated and characterized inside a nitrogen glovebox in order to avoid air exposure and unintentional doping effects.

Electrical Characterization: Electrical measurements were conducted in a nitrogen atmosphere at room temperature, using an Agilent B2902A parameter analyzer, while the transistor operating parameters were extracted using the gradual channel approximation model. ${ }^{[38]}$

Surface Characterization: Surface topography images and height distributions were obtained using the Agilent 5500 SPM atomic force microscope operating in tapping-mode. Budget Sensors Tap300Al-G silicon probes were used, with a spring constant of $40 \mathrm{Nm}^{-1}$ and a probe-radius $<10$ nm. Lateral conductive atomic force microscopy measurements were carried out using the same Agilent 5500 SPM microscope, operating in contact-mode. To maximize the electrical conductivity, platinum coated Mikromasch CSC17/Pt probes were used in this case, with a spring constant of $0.06 \mathrm{Nm}^{-1}-0.4 \mathrm{Nm}^{-1}$ and a coated probe-radius $<30 \mathrm{~nm}$. A bias of $-5 \mathrm{~V}$ was applied to the sample electrode, while the conductive probe was held at ground, enabling holes injection from the probe and their extraction through the negative biased electrode. Image planarization and data analysis were performed using Gwyddion 2.38, OriginPro 9.1 and Matlab R2014a software. 


\section{F. Bottacchi et al. Small (2016), DOI: $\underline{\text { 10.1002/smll.201600922 }}$}

\section{Acknowledgements}

The work was funded by the People Programme (Marie Curie Actions) of the European Union's Seventh Framework Programme FP7/2007-2013/: "Polymer-carbon nanotubes active systems for photovoltaics" (POCAONTAS), grant agreement n 316633 .

Received: ((will be filled in by the editorial staff))

Revised: ((will be filled in by the editorial staff)) Published online: ((will be filled in by the editorial staff $)$ )

\section{References}

[1] S. Z. Bisri, J. Gao, V. Derenskyi, W. Gomulya, I. Iezhokin, P. Gordiichuk, A. Herrmann, M. A. Loi, Adv. Mater. 2012, 24, 6147.

[2] G. J. Brady, Y. Joo, S. Singha Roy, P. Gopalan, M. S. Arnold, Appl. Phys. Lett. 2014, 104, 083107.

[3] V. Derenskyi, W. Gomulya, J. M. S. Rios, M. Fritsch, N. Fröhlich, S. Jung, S. Allard, S. Z. Bisri, P. Gordiichuk, A. Herrmann, U. Scherf, M. A. Loi, Adv. Mater. 2014, 26, 5969.

[4] M. C. Gwinner, F. Jakubka, F. Gannott, H. Sirringhaus, J. Zaumseil, ACS Nano 2012, 6, 539.

[5] S. P. Schieß1, N. Fro, M. Held, F. Gannott, M. Schweiger, M. Forster, U. Scherf, J. Zaumseil, ACS Appl. Mater. Interfaces 2015, 7, 682.

[6] F. Bottacchi, L. Petti, F. Späth, I. Namal, G. Tröster, T. Hertel, T. D. Anthopoulos, Appl. Phys. Lett. 2015, 106, 193302.

[7] M. J. Shea, M. S. Arnold, Appl. Phys. Lett. 2013, 102, 2011.

[8] L. Petti, F. Bottacchi, N. Münzenrieder, H. Faber, G. Cantarella, C. Vogt, L. Büthe, I. Namal, F. Späth, T. Hertel, T. D. Anthopoulos, G. Tröster, In Electron Device Meeting (IEDM); 2014; pp. 26.4.1-26.4.4.

[9] C. Wang, J.-C. Chien, K. Takei, T. Takahashi, J. Nah, A. M. Niknejad, A. Javey, Nano Lett. 2012, 12, 1527.

[10] H. Chen, Y. Cao, J. Zhang, C. Zhou, Nat. Commun. 2014, 5, 4097.

[11] A. Vena, L. Sydänheimo, M. M. Tentzeris, L. Ukkonen, In Proceedings of the 43rd European Microwave Conference; 2013; pp. 9-12.

[12] F. Jakubka, C. Backes, F. Gannott, U. Mundloch, F. Hauke, A. Hirsch, J. Zaumseil, ACS Nano 2013, 7, 7428.

[13] D. Stich, F. Späth, H. Kraus, A. Sperlich, V. Dyakonov, T. Hertel, Nat. Photonics 2013, 8, 


\section{F. Bottacchi et al. Small (2016), DOI: $\underline{10.1002 / \text { smll.201600922 }}$}

139.

[14] N. Rouhi, D. Jain, P. J. Burke, ACS Nano 2011, 5, 8471.

[15] Q. Cao, S. Han, G. S. Tulevski, Y. Zhu, D. D. Lu, W. Haensch, Nat. Nanotechnol. 2013, 8, 180.

[16] H. Liu, D. Nishide, T. Tanaka, H. Kataura, Nat. Commun. 2011, 2, 309.

[17] M. S. Arnold, A. a Green, J. F. Hulvat, S. I. Stupp, M. C. Hersam, Nat. Nanotechnol. 2006, 1,60 .

[18] J. Gao, M. Kwak, J. Wildeman, A. Herrmann, M. A. Loi, Carbon N. Y. 2011, 49, 333.

[19] J.-Y. Hwang, A. Nish, J. Doig, S. Douven, C.-W. Chen, L.-C. Chen, R. J. Nicholas, J. Am. Chem. Soc. 2008, 130, 3543.

[20] A. Nish, J.-Y. Hwang, J. Doig, R. J. Nicholas, Nat. Nanotechnol. 2007, 2, 640.

[21] H. Ozawa, N. Ide, T. Fujigaya, Y. Niidome, N. Nakashima, Chem. Lett. 2011, 40, 239.

[22] P. Finnie, J. Ding, Z. Li, C. T. Kingston, J. Phys. Chem. C 2014, 118, 30127.

[23] Z. Li, J. Ding, P. Finnie, J. Lefebvre, F. Cheng, C. T. Kingston, P. R. L. Malenfant, Nano Res. 2015, 8, 2179.

[24] S. Kumar, J. Y. Murthy, M. A. Alam, Phys. Rev. Lett. 2005, 95, 3.

[25] C. Kocabas, N. Pimparkar, O. Yesilyurt, S. J. Kang, M. A. Alam, J. A. Rogers, Nano Lett. 2007, 7, 1195.

[26] D. Sun, M. Y. Timmermans, Y. Tian, A. G. Nasibulin, E. I. Kauppinen, S. Kishimoto, T. Mizutani, Y. Ohno, Nat. Nanotechnol. 2011, 6, 156.

[27] N. Pimparkar, C. Kocabas, S. J. Kang, J. Rogers, M. A. Alam, IEEE Electron Device Lett. 2007, 28, 593.

[28] N. Pimparkar, Q. Cao, S. Kumar, J. Y. Murthy, J. Rogers, M. A. Alam, Electron Device Lett. IEEE 2007, 28, 157.

[29] G. E. Pike, C. H. Seager, Phys. Rev. B 1974, 10, 1421.

[30] S. Hunter, T. D. Anthopoulos, Adv. Mater. 2013, 25, 4320.

[31] W. S. Hu, Y. T. Tao, Y. F. Chen, C. S. Chang, Appl. Phys. Lett. 2008, 93.

[32] M. Toader, H. Fiedler, S. Hermann, S. E. Schulz, T. Gessner, M. Hietschold, Nanoscale Res. Lett. 2013, 8, 24.

[33] F. Jakubka, S. P. Schieß1, S. Martin, J. M. Englert, F. Hauke, A. Hirsch, J. Zaumseil, ACS Macro Lett. 2012, 1, 815.

[34] F. Schöppler, C. Mann, T. C. Hain, F. M. Neubauer, G. Privitera, F. Bonaccorso, D. Chu, A. C. Ferrari, T. Hertel, J. Phys. Chem. C 2011, 115, 14682.

[35] J. K. Streit, S. M. Bachilo, S. Ghosh, C.-W. Lin, R. B. Weisman, Nano Lett. 2014, 14, 1530.

[36] K. Liu, J. Deslippe, F. Xiao, R. B. Capaz, X. Hong, S. Aloni, A. Zettl, W. Wang, X. Bai, - 23 - 
S. G. Louie, E. Wang, F. Wang, Nat. Nanotechnol. 2012, 7, 325.

[37] See supplementary material at [URL will be inserted by Wiley] for further experimental details and data, .

[38] S. M. Sze, K. K. NG, Physics of Semiconductor Devices; Wiley New York, 2007.

[39] C. M. Aguirre, P. L. Levesque, M. Paillet, F. Lapointe, B. C. St-Antoine, P. Desjardins, R. Martel, Adv. Mater. 2009, 21, 3087.

[40] W. Kim, A. Javey, O. Vermesh, O. Wang, Y. M. Li, H. J. Dai, Nano Lett. 2003, 3, 193.

[41] T. D. Anthopoulos, G. C. Anyfantis, G. C. Papavassiliou, D. M. De Leeuw, Appl. Phys. Lett. 2007, 90, 89.

[42] Q. Cao, M. Xia, C. Kocabas, M. Shim, J. A. Rogers, S. V. Rotkin, Appl. Phys. Lett. 2007, $90,023516$. 


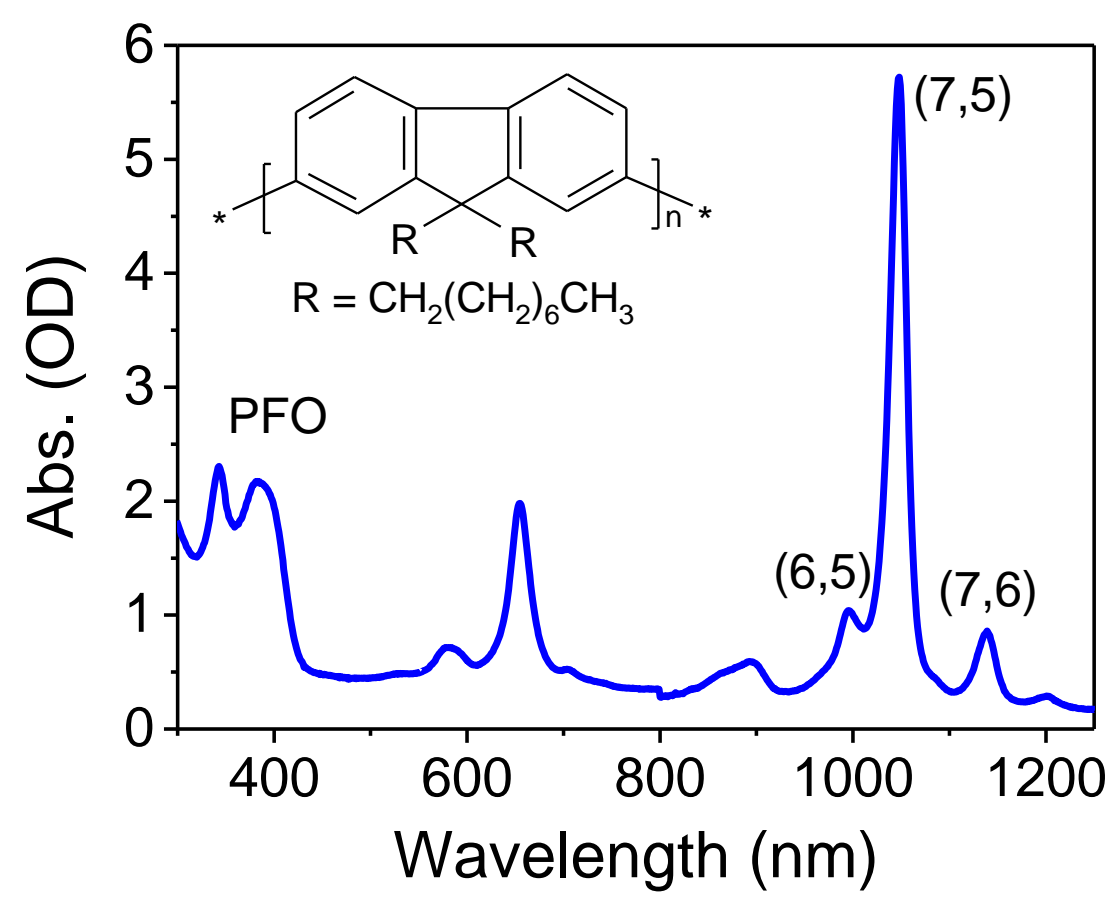

Figure 1. Absorption spectrum of the polymer-sorted $(7,5)$ SWNT dispersion in chlorobenzene. The sharp peaks at $\lambda=1050 \mathrm{~nm}$ and $\lambda=655 \mathrm{~nm}$, respectively, correspond to the first and second excitonic transitions of the $(7,5)$ nanotube. The absorption in the range of $340-380 \mathrm{~nm}$ corresponds to the polymer PFO. Two additional minor peaks are visible at $995 \mathrm{~nm}$ and $1140 \mathrm{~nm}$, which may be due to residual amounts of $(6,5)$ and $(7,6)$ semiconducting SWNTs, respectively. No absorption is present in the metallic SWNTs range $(450-550 \mathrm{~nm})$. Inset: Chemical structure of PFO. 

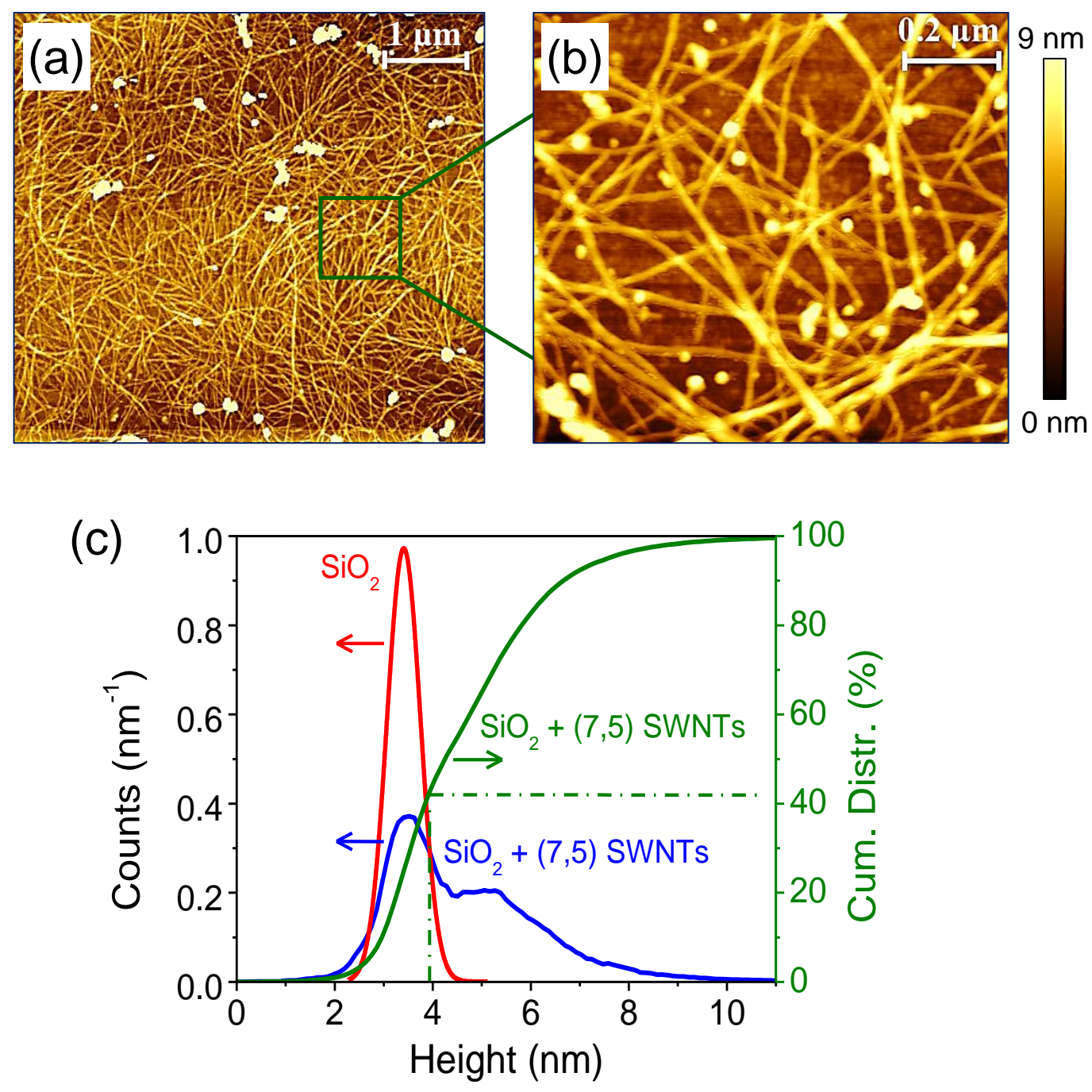

Figure 2. (a) AFM topography image, obtained in tapping-mode, of the as-spun polymer-sorted $(7,5) \mathrm{SWNT}$ network on the $\mathrm{SiO}_{2}$ gate dielectric surface. (b) Zoomed-in area of the image in (a), where bundles and SWNT aggregates are also visible. (c) Normalized height counts distributions of bare $\mathrm{SiO}_{2}$ and of $(7,5)$ SWNTs on $\mathrm{SiO}_{2}$ substrate. Corresponding height cumulative distribution of $(7,5) \mathrm{SWNT}$ on $\mathrm{SiO}_{2}$ substrate from which the relative coverage of $(7,5) \mathrm{SWNTs}$ and of $\mathrm{SiO}_{2}$ has been determined. 
(a)

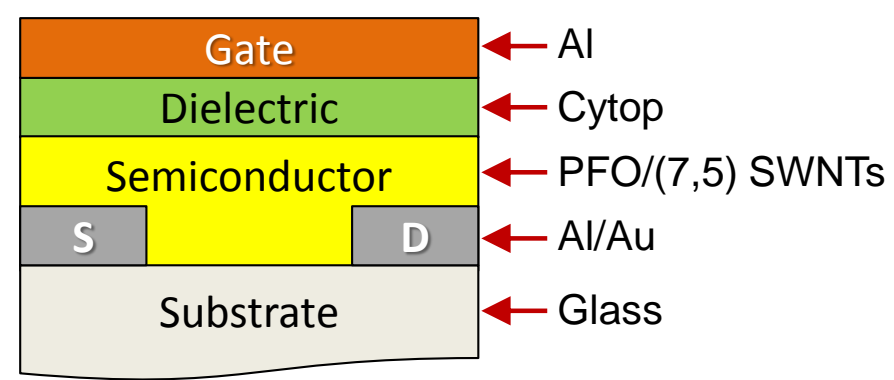

(b)

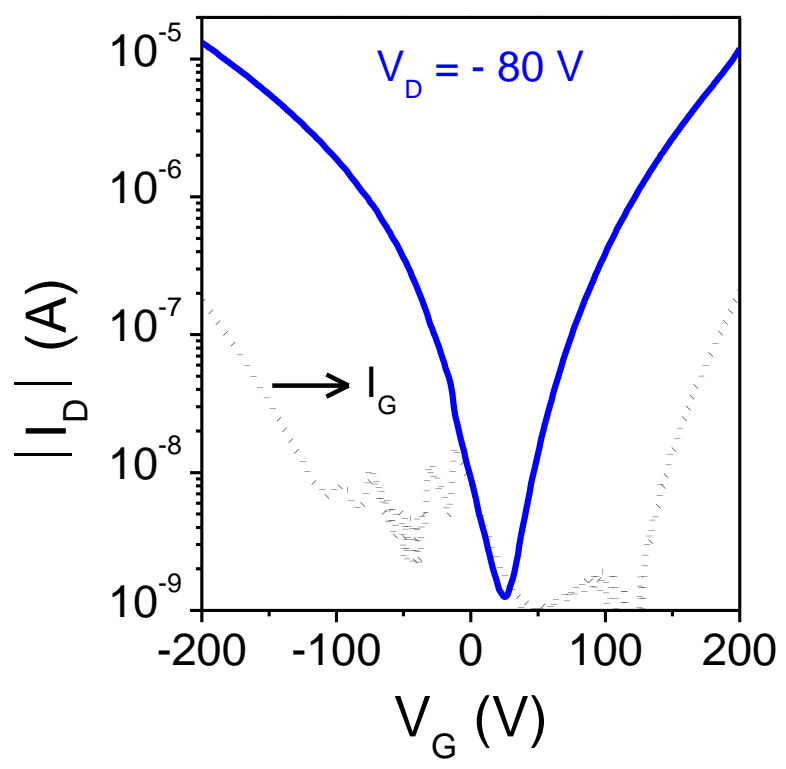

(c)



Figure 3. (a) Schematic of the top-gate field-effect transistor fabricated on a glass substrate. (b) Transfer characteristic of a polymer-sorted $(7,5)$ SWNTs top-gate transistor with $\mathrm{L}_{\mathrm{C}} / \mathrm{W}_{\mathrm{C}}$ of 50 $\mu \mathrm{m} / 1000 \mu \mathrm{m}$. The device shows ambipolar behavior with balanced hole and electron currents. (c) Corresponding transistor output characteristics. 
(a)

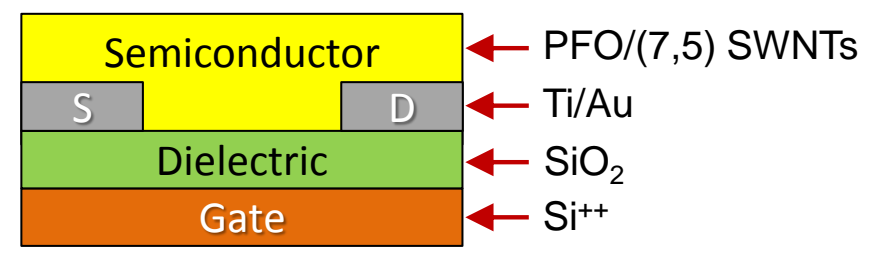

(b)

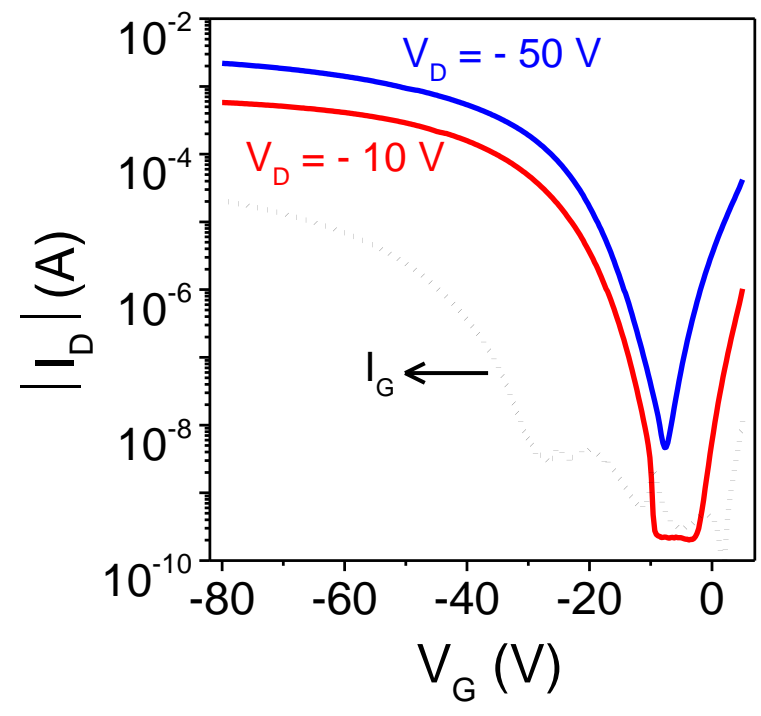

(c)

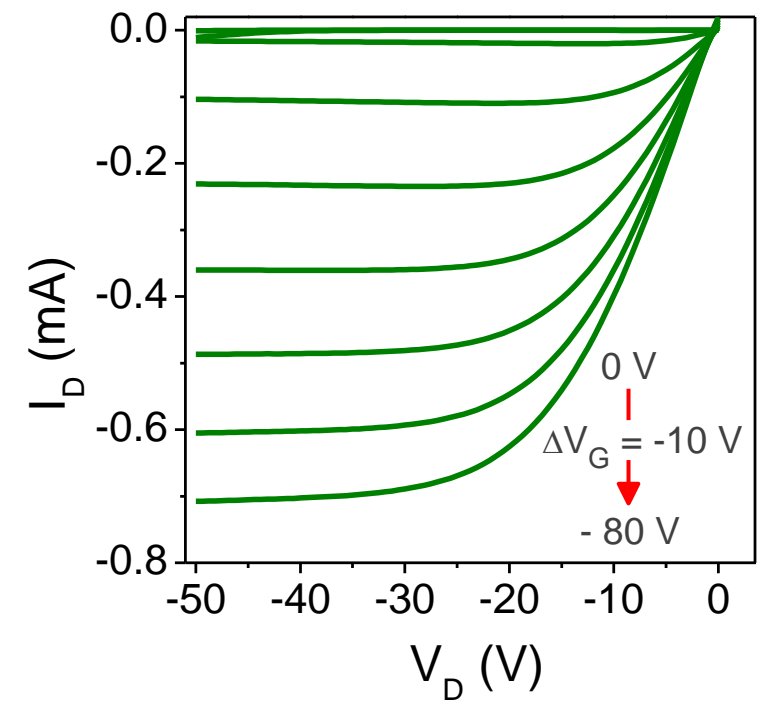

Figure 4. (a) Schematic of the bottom-gate field-effect transistor fabricated on a $\mathrm{Si}^{++} / \mathrm{SiO}_{2}$ substrate. (b) Transfer characteristic of a polymer-sorted (7,5) SWNTs bottom-gate transistor with $\mathrm{L}_{\mathrm{C}} / \mathrm{W}_{\mathrm{C}}$ of $20 \mu \mathrm{m} / 10000 \mu \mathrm{m}$. (c) Corresponding transistor output characteristics. 


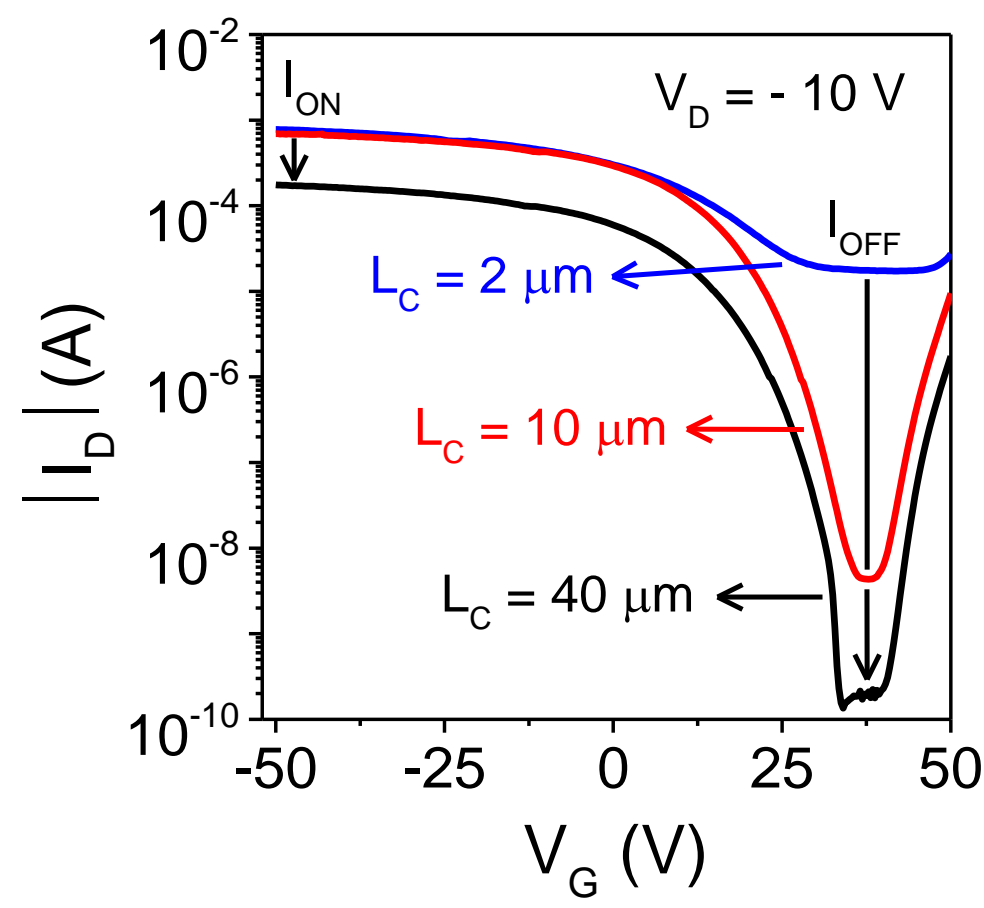

Figure 5. Transfer characteristics of three polymer-sorted $(7,5)$ SWNTs bottom-gate field-effect transistors with a constant $\mathrm{W}_{\mathrm{C}}$ of $10 \mathrm{~mm}$ and different $\mathrm{L}_{\mathrm{C}}(2 \mu \mathrm{m}, 10 \mu \mathrm{m}$, and $40 \mu \mathrm{m})$. All devices show predominant p-type behavior with similar ION currents but different IOFF currents. 
(a)

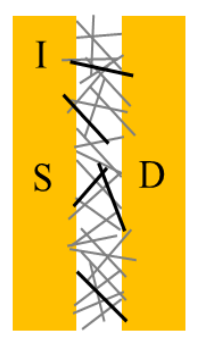

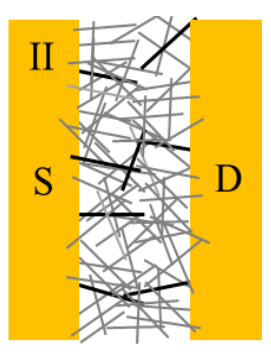

(c)

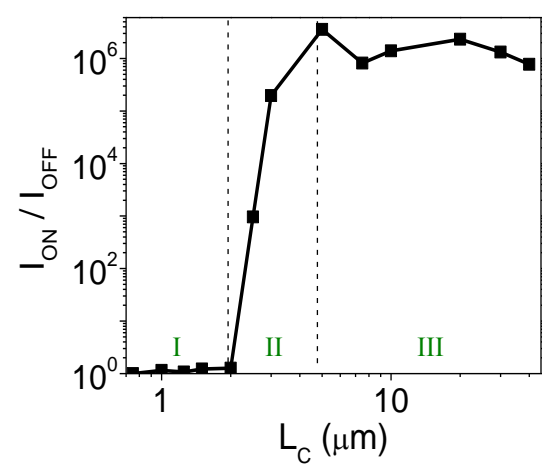

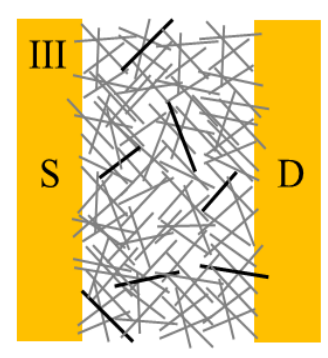

(b)

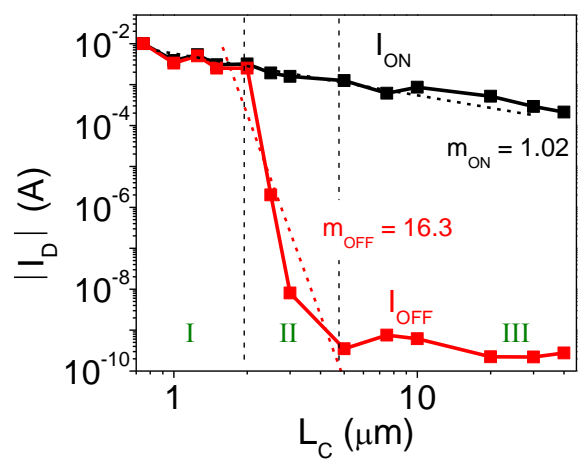

(d)

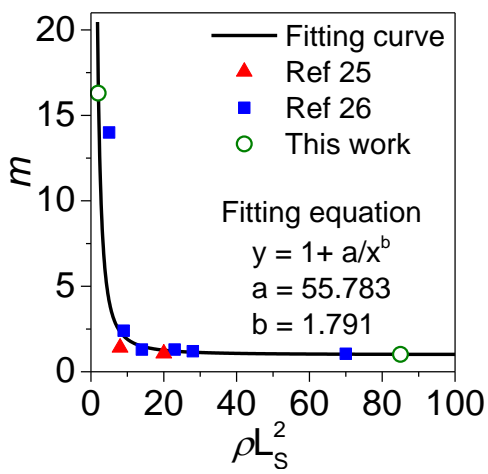

Figure 6. (a) Schematic of the SWNT network in the direct conduction regime (I), percolation regime (II), and thermally-excited charge transport regime (III). Grey sticks correspond to the vast majority of semiconducting SWNTs, while black sticks correspond to the few metallic SWNTs that can still form conduction paths in (I) and (II), but not in (III). (b) Channel ONcurrent (IoN) and OFF-current (IOFF) as a function of $\mathrm{L}_{\mathrm{C}}$, measured at $\mathrm{V}_{\mathrm{D}}=-10 \mathrm{~V}$ and $\mathrm{V}_{\mathrm{G}}=-50$ $V$. Current values in (b) have been averaged over four devices for each $L_{C}$ to account for deviceto-device parameter variability; $m_{\text {off }}$ has been obtained from the fitting line (red dash line) of the percolation region (II), while $m_{o n}$ from the fitting line (black dash line) of the full range. (c) Corresponding current ON/OFF ratio as a function of $\mathrm{L}_{\mathrm{C}}$. (d) Experimental curve of $m\left(\rho \mathrm{Ls}^{2}\right)$ obtained by fitting the data from references 25 and 26. Coverage values have been obtained from our experimental $m$ values and by using the fitting curve. 
(a)

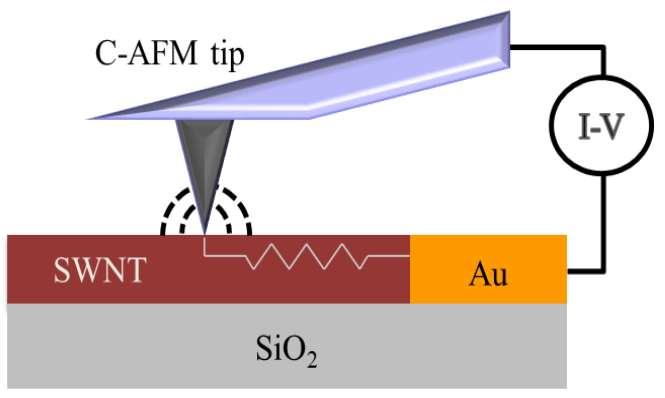

(b)

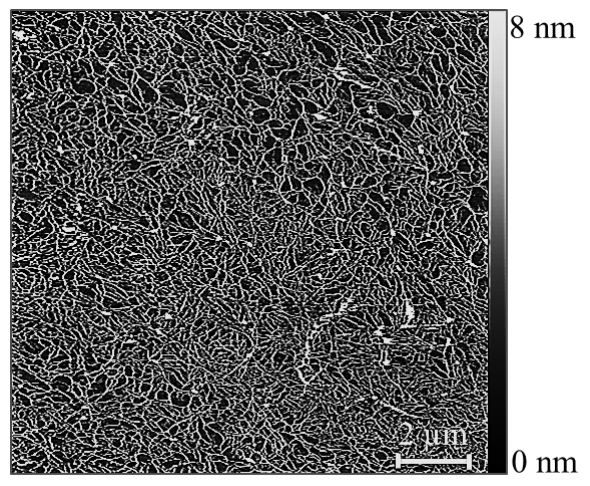

(d)

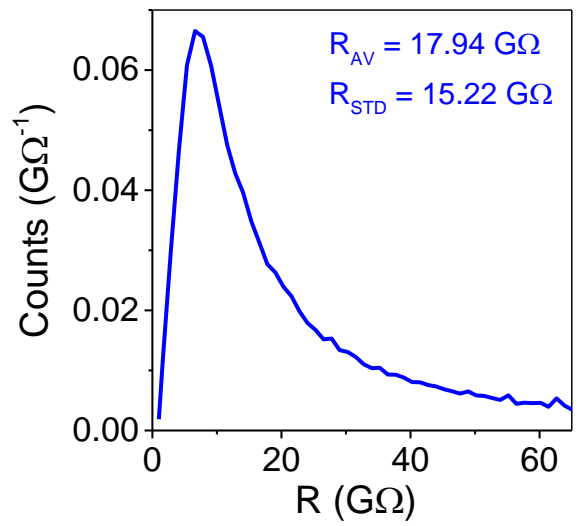

(c)

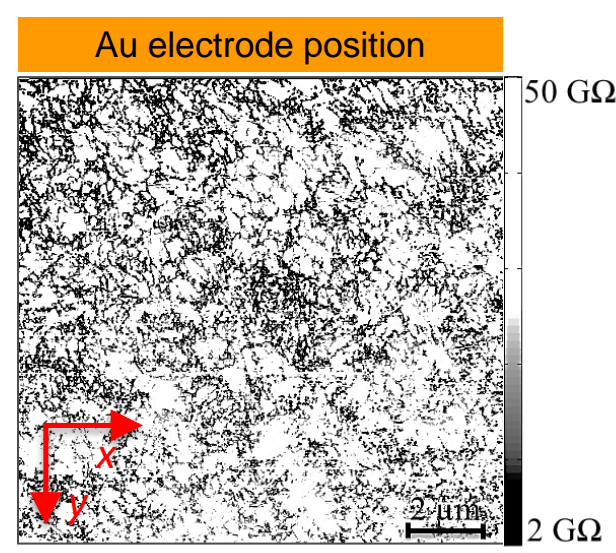

(e)

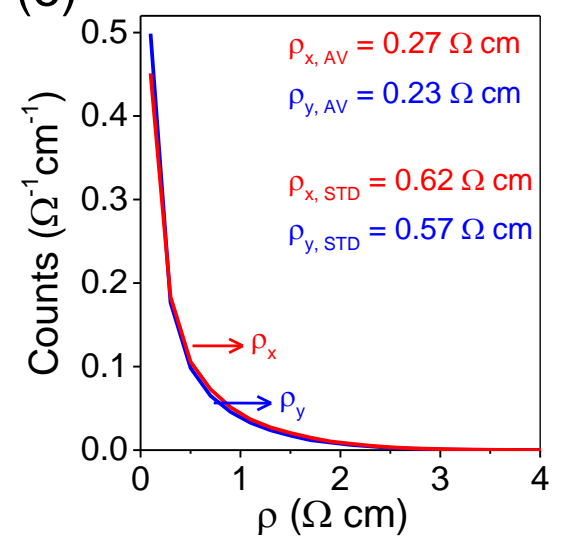

Figure 7. (a) Schematic representation of the lateral conductive AFM (C-AFM) setup operating in contact-mode. (b) C-AFM topography map obtained in contact-mode for an as-spun polymersorted $(7,5) \mathrm{SWNT}$ network deposited on $\mathrm{Si}^{++} / \mathrm{SiO}_{2}$. (c) Corresponding C-AFM resistance map. (d) Corresponding SWNT network resistance distribution, with average and standard deviation values. (e) Corresponding SWNT network resistivity distributions along $x$ and $y$ directions, with average and standard deviation values. 
F. Bottacchi et al. Small (2016), DOI: $\underline{10.1002 / \text { smll.201600922 }}$

\title{
Supporting Information
}

\section{Nanoscale Charge Percolation Analysis in Polymer-Sorted (7,5) Single-Walled Carbon Nanotube Networks}

\author{
Francesca Bottacchi $^{1}$, Stefano Bottacchi ${ }^{2}$, Florian Späth ${ }^{3}$, Imge Namal ${ }^{3}$, Tobias Hertel ${ }^{3}$, and \\ Thomas D. Anthopoulos ${ }^{1 *}$ \\ ${ }^{[1]}$ Department of Physics and Centre for Plastic Electronics, \\ Blackett Laboratory, Imperial College London, London SW7 2BW, United Kingdom \\ E-mail: thomas.anthopoulos@imperial.ac.uk

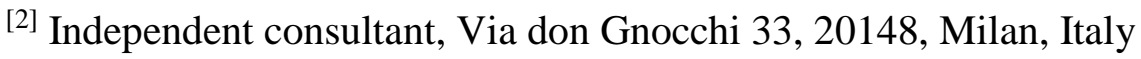 \\ ${ }^{[3]}$ Institute of Physical and Theoretical Chemistry, Faculty of Chemistry and Pharmacy \\ Julius-Maximilian University Würzburg, Am Hubland, 97074 Würzburg, Germany
}

\section{S1. (7,5) SWNT Length Characterization with AFM}

A detailed length characterization of the $(7,5)$ SWNTs has been performed on an atomically-flat $\mathrm{SiO}_{2}$ substrate with AFM in tapping-mode. In order to clearly distinguish single nanotubes, a diluted dispersion with an optical density (OD) of 1 has been used to measure the nanotubes lengths (Figure S1). Figure S2 shows the topography image of the $(7,5)$ SWNT network, where single carbon nanotubes are clearly visible on $\mathrm{SiO}_{2}$ surface. From this AFM topography image 239 single nanotubes have been manually counted and measured using Gwyddion software, obtaining an average tube length of $0.41 \mu \mathrm{m}$. Figure S3 shows the correspondent length distribution. It is important to observe that the nanotube lengths are very broadly distributed between $0.1 \mu \mathrm{m}$ and $1.5 \mu \mathrm{m}$, thus making it very difficult to choose the most representative length to use further in our analysis. Moreover, the presence of very short nanotubes can be attributed to the long sonication time necessary to achieve a high level of dispersion, which is known to damage and break nanotubes. For the purpose of our percolation study, the longest 


\section{F. Bottacchi et al. Small (2016), DOI: $\underline{10.1002 / \mathrm{smll.201600922}}$}

nanotubes certainly dominate the transport in the FET channel over the shortest ones. To account for this we have obtained the square-length weighted abundance distribution, in order to estimate the effective length contributing to percolation, which takes into account the bias of percolation towards longer nanotubes in a sample with length-dispersion such as the one used in this study (Figure S2). Finally, the impact of effective nanotube length has been examined by changing its value within the experimentally determined distribution shown in Figure S3.

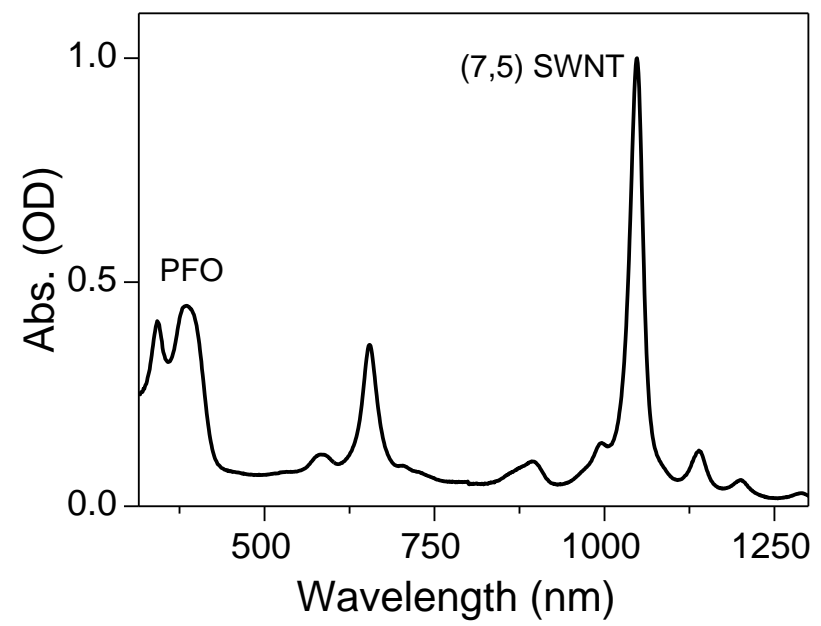

Figure S1: Absorption spectrum of the $\mathrm{PFO} /(7,5) \mathrm{SWNT}$ dispersion used to characterize the nanotube length.

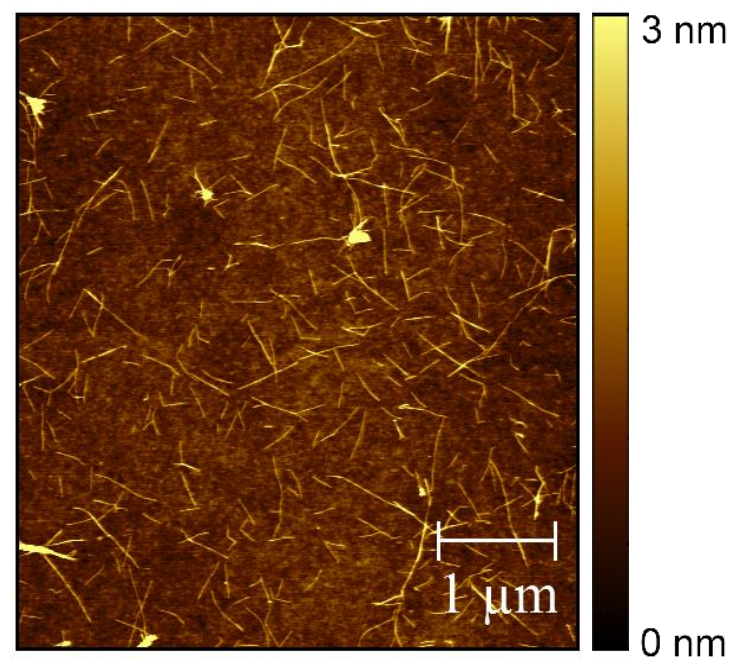

Figure S2: AFM topography image of a $(7,5)$ SWNT network spin-coated on $\mathrm{Si}^{++} / \mathrm{SiO}_{2}$ wafer. 


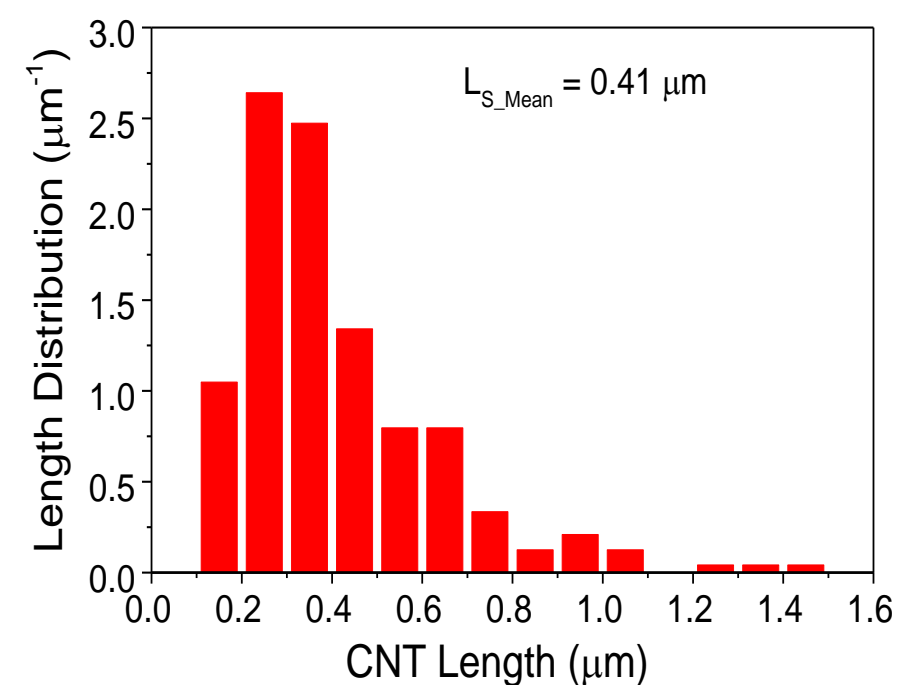

Figure S3: Histogram showing the normalized distribution of the $(7,5)$ SWNT length with the correspondent mean value taken from the topography image shown in Figure $\mathbf{S 2}$.

\section{S2. $(7,5)$ SWNT Film Orientation from C-AFM Analysis}

From the analysis of the C-AFM resistivity map along $\mathrm{x}$ and $\mathrm{y}$ directions, it is possible to assess if the network is isotropic or if nanotubes are orientated along a certain preferential direction. This assessment is based on the fact that in a carbon nanotube, transport along the tube axis is much more effective then transport perpendicular to the tube axis, thus resistivity along the tube direction, where ballistic-like transport occurs, has a very small mean value and also a narrow distribution with a small standard deviation. On the other hand, resistivity perpendicular to the tube axis, where hopping-like transport occurs, is characterized by a higher mean value and a broader distribution, meaning a higher standard deviation. By comparing resistivities along two perpendicular directions, once the electrode location is fixed, it is possible to determine the preferential orientation of the network, if any. Figure S4(a) shows the AFM image obtained in contact-mode of a partially aligned film of polymer-sorted $(7,5)$ SWNT, blade-coated along $y$ direction. Figure $\mathbf{S 4 ( b )}$ shows the corresponding resistivity distributions. The fact that the 


\section{F. Bottacchi et al. Small (2016), DOI: $\underline{10.1002 / \text { smll.201600922 }}$}

average value and standard deviation of resistivity along $y$ is smaller than along $x$, is indicative of a partial alignment along y direction.

(a)

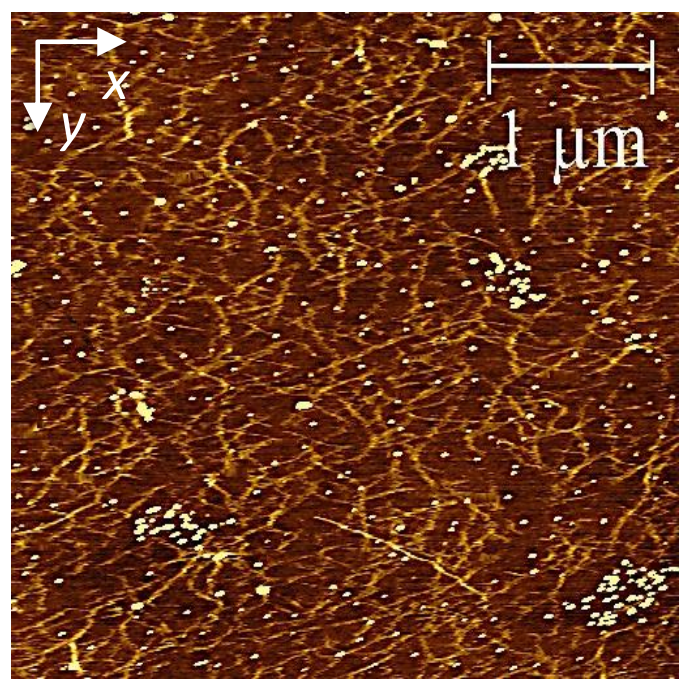

(b)

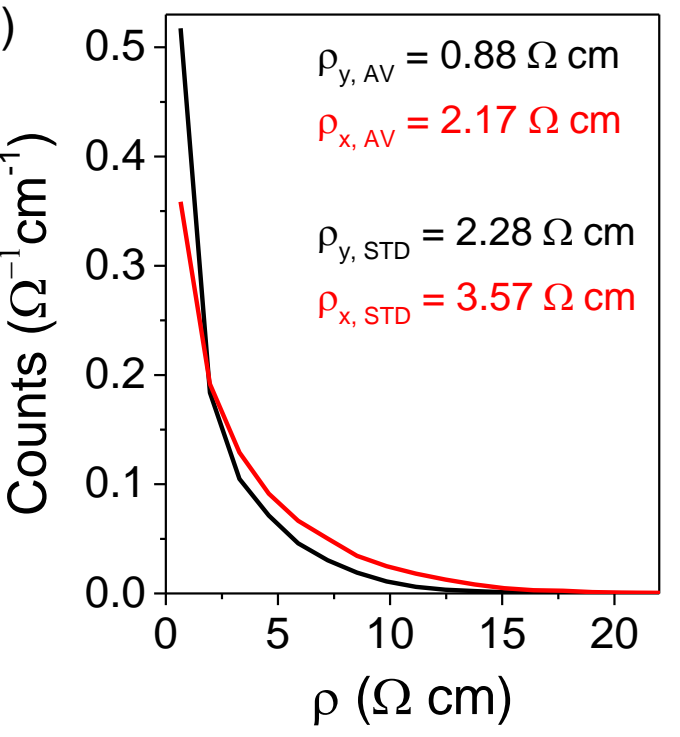

Figure S4: (a) C-AFM topography map obtained in contact-mode for a partially aligned polymer-sorted $(7,5) \mathrm{SWNT}$ film blade-coated along $y$ direction on $\mathrm{Si}^{++} / \mathrm{SiO}_{2}$. (b) Corresponding SWNT film resistivity distributions along $x$ and $y$ directions, with average and standard deviation values. 